\title{
Guillain-Barré syndrome in the COVID-19 era: another occasional cluster?
}

\author{
Laurent Tatu ${ }^{1,2}$ (1) $\cdot$ Sandra Nono ${ }^{3} \cdot$ Simone Grácio ${ }^{4} \cdot$ Serdar Koçer $^{4}$
}

Received: 2 June 2020 / Accepted: 16 June 2020 / Published online: 23 June 2020

c) Springer-Verlag GmbH Germany, part of Springer Nature 2020

\section{Dear Sirs,}

We read with interest the letter from Gigli et al. entitled 'Guillain-Barré syndrome in the COVID-19 era: just an occasional cluster?' [1]. The authors reported an unusual cluster of seven patients affected by Guillain-Barré syndrome (GBS) in an Italian region (Friuli Venezia-Giulia), which coincided with the descending curve of the COVID19 pandemic. All patients had a negative SARS-CoV-2 nasopharyngeal swab and all but one had negative IgM and IgG SARS-CoV-2 serology. The authors wondered if similar clusters had been observed elsewhere.

In our region, which spans the French-Swiss border, we have also been surprised by an abnormal number of GBS cases during the March-April 2020 period corresponding to the peak of the COVID-19 pandemic. In this area, patients with GBS in the acute and subacute stages are admitted to one of two hospitals, Centre Hospitalier Universitaire de Besançon (France) and Hôpital du Jura (Porrentruy-Delémont-Switzerland). Using the same procedure as Gigli et al., we re-examined the typology of the disease.

This Letter to the Editor refers to the original publication: https:// link.springer.com/content/pdf/10.1007/s00415-020-09911-3

A reply to this letter can be read here: https://doi.org/10.1007/ s00415-020-10006-2

\section{Laurent Tatu}

laurent.tatu@univ-fcomte.fr

1 Department of Neuromuscular Diseases, CHRU Besançon, University of Franche-Comté, 3 boulevard Fleming, 25000 Besançon, France

2 Department of Anatomy, University of Franche-Comté, Besançon, France

3 Department of Neurology, CHRU Besançon, Besançon, France

4 Department of Rehabilitation, Hôpital du Jura, Porrentruy, Switzerland
In the public health crisis of March-April 2020, we encountered an unusually high number of GBS cases, admitting seven patients. In each of the previous 3 years, in the same time period and region, the number of GBS cases ranged from 0 to 2 .

One of our cases was related to an Epstein-Barr viral infection. The clinical, biological, and electrophysiological characteristics of the other six patients are detailed in Table 1. The SARS-CoV-2 nasopharyngeal swab and SARSCoV-2 serology were negative in all six patients. Patient 3 developed the first GBS signs 14 days after an influenza vaccination, but his biological evaluation showed a slight hepatic cytolysis as well as a positive hepatitis E serology. Even if the patient expressed no clinical signs of hepatitis, a relationship between GBS and hepatitis E could be argued [2]. No other potential etiology was found in our patients (Table 1).

Only one patient (patient 3) had an acute motor-sensory axonal neuropathy (AMSAN); acute inflammatory demyelinating polyneuropathy (AIDP) was found in the other five patients. All patients were treated with intravenous immunoglobulins. Three of them were admitted to an intensivecare unit. The neurological progression was favorable in five cases. Two of the patients (patients 1 and 2) relapsed, one of whom died from a severe acute respiratory syndrome. One patient (patient 6) had a two-stage evolution with a sudden worsening (tetraparesis and cranial nerve paralyses) 4 days after symptom onset, suggesting a Bickerstaff-like encephalitis.

Extrapulmonary complications of COVID-19 frequently include the nervous system, due to a particular tropism of SARS-CoV-2 [3]. Some authors report a possible correlation between acute symptomatic COVID-19 infection and GBS [4, 5]. Nevertheless, the issue raised by Gigli's cases and those in this series is different: an abnormally high frequency of GBS amid the SARS-CoV-2 pandemic in patients without a COVID infection. 


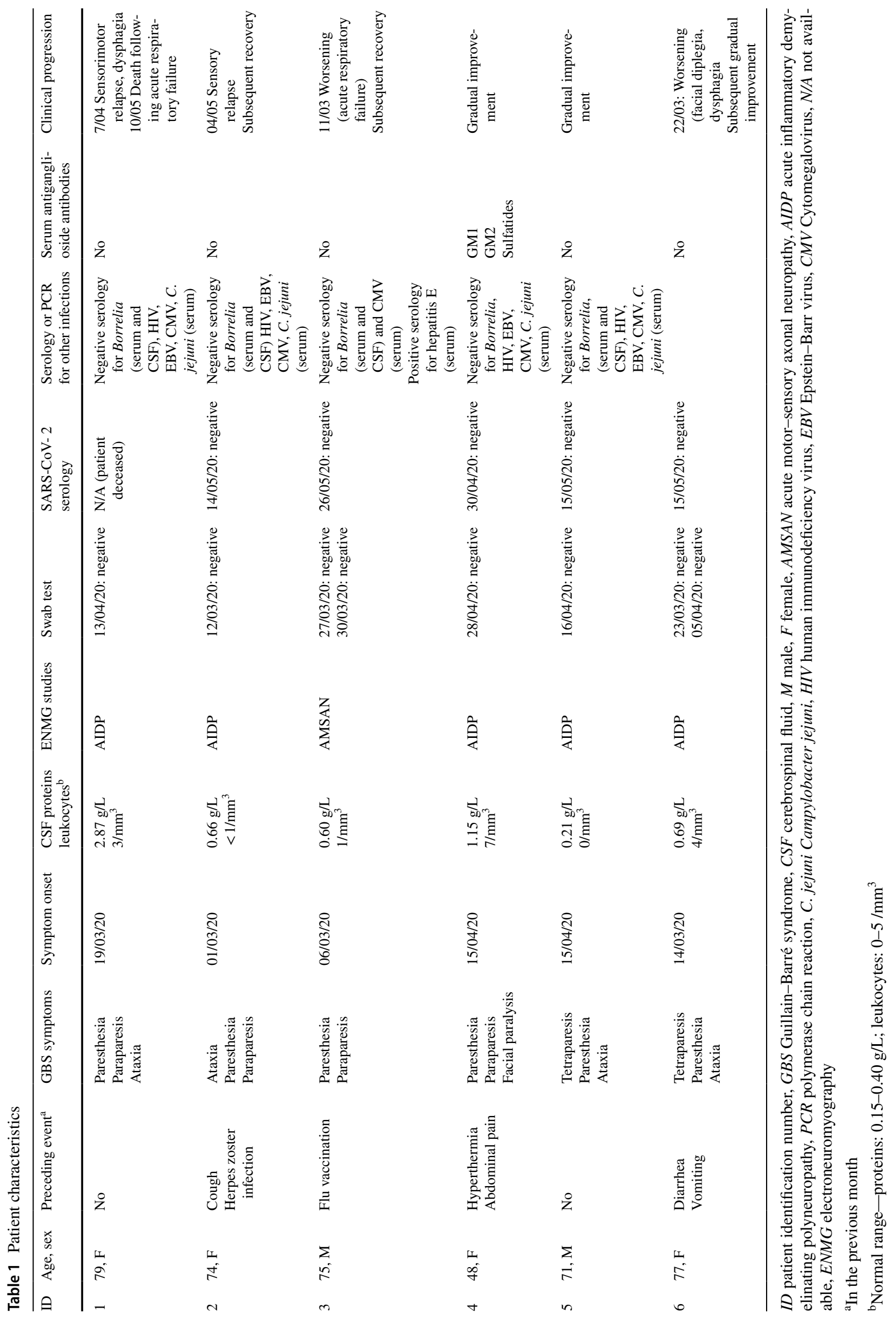


The specificity and sensitivity of swab-test and serologies are better known now than at the time of writing for Gigli et al., as is the curve of the humoral immune response to this new virus. Nevertheless, as expressed by Gigli et al., it is possible that asymptomatic or paucisymptomatic infections may not develop an antibody response sufficient enough to be detected [1]. Another hypothesis is that an asymptomatic contact with SARS-CoV-2 could be a precipitating factor for the preceding event, causing an immunologic cascade that leads to GBS. Such a concomitant immunologic mechanism could potentially be observed in other autoimmune diseases.

\section{Compliance with ethical standards}

Conflicts of interest The authors declare that they have no conflict of interest.

Ethical approval No specific ethics approval was required.

\section{References}

1. Gigli GL, Bax F, Marini A, Surcinelli A, Valente M (2020) Guillain-Barré syndrome in the COVID-19 era: just an occasional cluster? J Neurol. https://doi.org/10.1007/s00415-020-09911 -3 (Online ahead of print)

2. Liu H, Ma Y (2020) Hepatitis E virus-associated Guillain-Barre syndrome: revision of the literature. Brain Behav 10(1):e01496

3. Li YC, Bai WZ, Hashikawa T (2020) The neuroinvasive potential of SARS-CoV2 may play a role in the respiratory failure of COVID-19 patients. J Med Virol. https://doi.org/10.1002/ jmv. 25728

4. Zhao H, Shen D, Zhou H, Liu J, Chen S (2020) Guillain-Barré syndrome associated with SARS-CoV-2 infection: causality or coincidence? Lancet Neurol 19(5):383-384

5. Alberti P, Beretta S, Piatti M, Karantzoulis A, Piatti ML, Santoro P, Vigano M, Giovannelli G, Pirro F, Montisano DA, Appollonio I, Ferrarese C (2020) Guillain-Barré syndrome related to COVID19 infection. Neurol Neuroimmunol Neuroinflamm 7:e741. https ://doi.org/10.1212/NXI.0000000000000741 